\title{
DESAJUSTES DEL MOBILIARIO UNIVERSITARIO A LAS CARACTERÍSTICAS ANTROPOMÉTRICAS DE LOS ESTUDIANTES
}

\author{
MISMATCH OF THE UNIVERSITY FURNITURE TO THE ANTHROPOMETRIC \\ CHARACTERISTICS OF THE STUDENTS
}

\author{
Paula Martha Veliz Terry ${ }^{1}$ \\ Carlos Manuel Escobar Galindo
}

\begin{abstract}
Resumen: Los estudiantes universitarios permanecen largo tiempo sentados en diferentes ambientes, utilizando por lo general mobiliario unipersonal, los cuales si no se encuentran acorde a sus características antropométricas, podrían generar problemas a su salud. El objetivo de este estudio es determinar el nivel de desajuste del mobiliario a las características antropométricas de los estudiantes de una universidad nacional. Se utilizó un diseño observacional, descriptivo, relacional y de corte transversal, cuya muestra estuvo conformada por 194 estudiantes. Los instrumentos aplicados fueron: ficha para la recolección de las medidas de las dimensiones antropométricas y ficha de medidas del mobiliario. Se encontró que las medidas de profundidad de asiento, altura de asiento y altura de tablero fueron en gran porcentaje no adecuadas, con un 66\%, 75\% y 97\% respectivamente. Por el contrario, la altura del respaldar muestra una tendencia inversa alcanzando un valor mayor en la categoría adecuado, con un 69\%, que en la categoría no adecuado, con un 31\%. Existió asociación entre género y mediciones antropométricas en las medidas de altura de asiento, respaldar y profundidad $(\mathrm{p}<0,05)$. Del total de observaciones, el 64,1\% de las medidas fueron no adecuadas, y el 34,9\% fueron adecuadas. Se concluyó que no hubo armonía proporcional evidente en los mobiliarios. Las mujeres tuvieron mayores problemas de adaptación en alturas y profundidades. Por lo tanto, los resultados mostraron que había una gran falta de coincidencia entre las medidas de los muebles y las dimensiones antropométricas de los estudiantes universitarios que pueden ser perjudiciales para su bienestar y rendimiento durante las clases.
\end{abstract}

Palabras clave: Ergonomía (DeCS), antropometría (DeCs), desordenes musculoesqueléticos (Mesh), mobiliario de clases (MesH).

Abstract: University students remain seated in different environments for a long time, generally using one-person furniture, which, if it is not in accordance with their anthropometric characteristics, may produce health problems. The objective was to determine the match level of the one-person furniture to the anthropometric characteristics of the students of a national university. Methods: Observational, descriptive, relational and cross-sectional design. The sample size consisted of 194 students. The instruments applied were: Sheet for the collection of measurements of anthropometric dimensions and Sheet for furniture measurements. The variables seat depth, seat height and board height showed a high trend towards the not adequate aspect, with a $66 \%, 75 \%$ and $97 \%$ respectively.

1Universidad Nacional Mayor de San Marcos. Lima, Perú. Correo electrónico: pvelizt@unmsm.edu.pe. Orcid: https://orcid.org/oooo-0002-7332-2727

${ }^{2}$ University of Nottingham. Nottingham, United Kingdom. Correo electrónico: manoergo@gmail.com, manuel.escobar@nottingham.ac.uk. Orcid: https://orcid.org/oooo-0001-7263-9215 
Conversely, the height of the backrest shows an inverse trend reaching a higher percentage in the adequate category, with a $69 \%$, than in the not adequate category, with a $31 \%$. There was an association between gender and anthropometric measurements in the measurements of seat height, backrest and depth ( $\mathrm{p}<0.05$ ). The one-person furniture, with a coupled board, is for the majority "Not suitable". From the total observations, $64.1 \%$ of the measures were not adequate, and $34.9 \%$ were adequate. It was conclude that a proportional harmony was not evident in the furniture. The women had greater problems of adaptation in heights and depths. Hence, the findings showed that there was a high mismatch between the measurements of the furniture and the anthropometric dimensions of university students that may be harmful to their wellbeing and performance during classes.

Keywords: Ergonomics, anthropometrics, musculoskeletal diseases, classroom furniture.

Recepción: 24.09.2020 / Revisión: 12.10.2020 / Aceptación: 25.11.2020

\section{Introducción}

El estudiante universitario por lo general recibe una formación que lo obliga a permanecer muchas horas consecutivas sentado en diferentes ambientes de estudio (aula, laboratorio, biblioteca, computadora), casa, y/o centros laborales. Los estudiantes pasan alrededor de un tercio del día en la universidad, de los cuales aproximadamente el 80\% del tiempo ocupan un mobiliario universitario (Hoque et al., 2014). Permanecer sentado por tiempos prolongados en mobiliarios no adecuados ocasiona un riesgo mayor, no solo de sufrir trastornos musculoesqueléticos (TME), sino problemas cardíacos entre otros daños a la salud (Parvez et al., 2014). El efecto acumulativo en el sistema musculoesquelético debido a las demandas externas producidas por el mobiliario, tiene un efecto que incrementa el riesgo de lesiones y de compresiones, los cuales se evidencian primero como molestias musculoesqueléticas (MME), que sumados a los ajustes posturales producto de la adecuación incrementan la carga estática de diversos segmentos como los de cuello, hombros y columna dorsolumbar, así como también la presión a nivel de los glúteos (Castellucci et al., 2019, Gutiérrez \& Apud, 1992, Párraga-Velásquez \& García-Zapata, 2014, Thariq et al., 2010).

Las instituciones universitarias equipan sus aulas, por lo general, con un mismo modelo de mobiliario, considerando su apariencia, calidad y costo para su adquisición, más no al estudiante quien le dará uso. Esta falta de criterios ergonómicos podría dañar la salud e interferir en el rendimiento académico de los estudiantes. Por tanto, los mobiliarios deben ser diseñados tomando en cuenta los principios de ergonomía (Pheasant \& Haslegrave, 2006). La ergonomía es la disciplina científica que busca adecuar el trabajo al hombre tomando en cuenta sus características físicas y mentales para mejorar su bienestar y desempeño ocupacional (Grandjean \& Kroemer, 1995).

Como antecedente, hay diferentes estudios realizados en mobiliario escolar donde se han encontrado grandes desajustes, evidenciando pobres posturas, incomodidad, y MME (Gutiérrez \& Apud, 1992, Parcells et al., 1999). Estas discrepancias trajeron a discusión la necesidad de tener que actuar sobre los mobiliarios escolares como herramienta básica para lograr un óptimo desempeño en los estudios. De igual manera, a nivel universitario donde el crecimiento biológico ya se detuvo, pueden encontrarse problemas severos de adecuación 
especialmente cuando la población es tan diversa como la del Perú (Asgari et al., 2019). Sin embargo, en la actualidad se comercializan carpetas, sillas y/o mesas de medidas similares, pero sin criterios ergonómicos en su diseño y confección. Diferentes investigadores concluyen que para un diseño óptimo del mobiliario, muebles y herramientas es imprescindible que se adecue a las características antropométricas de los usuarios (Castellucci et al., 2019, Castellucci et al., 2010, Párraga-Velásquez \& García-Zapata, 2014, Ramírez, 2006).

A nivel mundial, se han efectuado estudios antropométricos en diferentes países, algunos en población escolar, y en menor cuantía en población universitaria. En América Latina, países como Chile, Costa Rica, México, Colombia, Brasil, cuenta con estudios a gran escala, con respaldo gubernamental, pero no actualizados (Ávila et al., 2001). Sin embargo, en el Perú la única referencia antropométrica con fines ergonómicos es la del trabajador minero de altura, donde se describen las características de varones del centro del Perú (Ramírez, 2006). De acuerdo al estudio reciente publicado de la Universidad de Harvard, los peruanos son una de las poblaciones más bajas del mundo, en especial las mujeres (Asgari et al., 2019, NCD Risk Factor Collaboration [NCD-RisC], 2016), lo cual desde el punto de vista ergonómico puede resultar perjudicial para asegurar una buena adecuación del trabajo al hombre (Pheasant \& Haslegrave, 2006a).

Dentro de los estándares de legalidad para asegurar criterios de calidad académica en el ámbito universitario peruano, el Sistema Nacional de Evaluación, Acreditación y Certificación de la Calidad Educativa ([SINEACE], 2016) hace referencia en el estándar 28, sobre "equipamiento e infraestructura", que el programa de estudios tiene que contar con infraestructura (salones de clase, oficinas, laboratorios, talleres, equipamiento, etc.) y el equipamiento pertinente para su desarrollo, más no establece las características específicas que debería tener. Al no existir directivas precisas que regulen este estándar, las universidades adquieren el mobiliario estudiantil al mejor postor.

Diferentes estudios señalan que el mejor mobiliario escolar corresponde al de características unipersonales con mesa separada (Castellucci, et al., 2010, Gutiérrez \& Apud, 1992), sin embargo, en la actualidad las universidades cuentan con carpetas unipersonales con tablero acoplado al ser las de mayor comercialización, variando en su calidad, pero de medidas bastante similares. Esta situación se debe principalmente a la falta de criterios y especificaciones técnicas para adquirir un mobiliario adecuado. Como antecedente, el Instituto Nacional de Defensa de la Competencia y de la Propiedad Intelectual del Perú en sus Normas Técnicas de Equipamiento a Instituciones Educativas, establece exigencias y planos para la dotación de mobiliario del nivel inicial, primaria y secundaria, así como también para los docentes, cuya última actualización data del 2012 sin presentar cambio alguno (Normas Técnicas Peruanas sobre muebles, tecnología de la información, tortas de semillas oleaginosas y otra [NTP], 2012). Además, se debe agregar que, las especificaciones son estándar, sin sustento de estudios antropométricos y ergonómicos, no tomando en cuenta la variabilidad de la población.

Por esta razón, el presente estudio tiene como objetivo determinar el nivel de desajuste del mobiliario unipersonal con tablero acoplado, a las características antropométricas de los estudiantes universitarios y su asociación con el género, así como también establecer un 
perfil antropométrico base referencial para el dimensionamiento del mobiliario universitario.

\section{Materiales y métodos}

De acuerdo con el problema planteado y el objetivo general definido, esta investigación siguió un diseño observacional o no experimental, descriptivo y de corte transversal.

Se calculó el tamaño de la muestra considerando una proporción esperada de 70\% de individuos en mobiliarios no adecuados (Hoque et al., 2014) con un nivel de confianza del 95 $\%$ y una precisión del $5 \%$ tomando en cuenta una población de 427 estudiantes de posgrado, obteniendo un total de 184 participantes como mínimo. El número total de estudiantes que participaron en el estudio fue de 194 excediendo el mínimo recomendado.

Se consideró tomar a una población de una Universidad Nacional, por la afluencia de universitarios, excluyendo a aquellos que tuvieran alguna discapacidad o alteración física o en situación gestacional avanzada. Para recolectar información necesaria para desarrollar el perfil antropométrico se elaboraron fichas para las mediciones antropométricas, siguiendo las recomendaciones de la Sociedad Internacional de Antropometría (ISAK) (Norton et al., 2000) y de la ISO 7250 (2017). Las medidas consideradas para elaborar el perfil antropométrico fueron las relacionadas al diseño de una silla y mesa estándar. Las medidas consideradas fueron tomadas en bipedestación y sedestación: La única medida en bípedo fue la estatura. Para las medidas en sedente se consideraron: (1) ancho de cadera (AC), (2) distancia glúteo-poplítea (DGP), (3) altura poplítea, (AP) (4) distancia escapulo asiento (DEA) y (5) altura del codo asiento (CA). Para asegurar la confiabilidad y exactitud de los datos, todas las medidas fueron tomadas con un antropómetro estándar marca Holtain calibrado con un error de precisión de $1 \mathrm{~mm}$. Todas las medidas fueron tomadas por los autores, quienes son especialistas en ergonomía, con apoyo de estudiantes de terapia ocupacional previamente entrenados, quienes adoptaron el rol de anotadores.

Se utilizó una segunda ficha para recolectar la información sobre el mobiliario. El mobiliario elegido para el trabajo fue un mobiliario unipersonal con tablero acoplado, de alto uso en los diferentes salones de la universidad. Se tomaron las medidas del mobiliario con ayuda de un flexómetro calibrado de fábrica. Para la toma de medidas, se convocó a los participantes, por medio de folletos informativos y correos electrónicos con apoyo de la base de datos de la universidad. Durante la selección se les informó los objetivos del estudio, y si estaban de acuerdo en participar, se les solicitó su consentimiento informado. Las medidas fueron tomadas con apoyo de un anotador previamente entrenado. Los procedimientos para la toma de medidas fueron los que sugieren la norma ISO 7250 (2017). Las medidas en sedente se realizaron con ayuda de un banco regulable que facilita el ajuste a la altura de cada participante. Las mediciones que implican a participantes damas, fueron realizadas por la investigadora, por consideraciones éticas.

Para la toma de medidas de los mobiliarios, se consideró la probable variabilidad entre ellos, por lo que se tomaron medidas a seis mobiliarios similares en diferentes salones (4) para evitar algún sesgo de variabilidad. El promedio de las medidas finales fue el representativo y redondeado a un número entero (ver figura 1). 
Los niveles de adecuación se determinaron a partir de las ecuaciones recomendadas por Parvez et al. (2014) y Pheasant \& Haslegrave (2006a) en donde: ancho de asiento (AS)= $1,1 \mathrm{O}(\mathrm{AC})<=\mathrm{AS}<=1,30$ (AC); profundidad de asiento (PA) $=0,80(\mathrm{DGP})<=\mathrm{PA}<=0,95(\mathrm{DG})$; altura de asiento $(\mathrm{ASA})=(\mathrm{AP}+3 \mathrm{~cm}){ }^{*} \cos 30<=\mathrm{ASA}<=(\mathrm{AP}+3 \mathrm{~cm}) *$ cos $5 \mathrm{y}$ altura del respaldo $(\mathrm{AR})=(\mathrm{EA}-5 \mathrm{~cm})<=\mathrm{AR}<=(\mathrm{EA}+5 \mathrm{~cm})$ y altura del tablero $(\mathrm{AT})=\mathrm{CA}<=\mathrm{AT}<=\mathrm{CA}+5$. Cuando los niveles no eran adecuados, entonces la variable obtuvo dos dimensiones: excede la medida y faltó medida. Dependiendo de la dimensión esta valoración tuvo diferentes connotaciones: muy ancho, estrecho para AS; muy alto, muy bajo para ASA, AR y AT, y muy profundo, poco profundo para PA. En caso la variable estuviera dentro del límite adecuado, fue clasificada como "adecuada". Para el análisis final se determinó la diferencia entre nivel "adecuado" y "no adecuado".

Todos los datos fueron ordenados en una plantilla de Microsoft office Excel (TM) para posteriormente ser procesados de acuerdo a la ecuación correspondiente a las medidas antropométricas. Para realizar la tabla con los perfiles antropométricos se siguieron los parámetros establecidos en la ISO 15535 (2012, p.15).

Finalmente, los resultados fueron codificados y posteriormente procesados en el paquete estadístico SpSS v.24 (IBM). Se utilizaron tablas de frecuencia y porcentajes para expresar todos los datos que corresponden a estadística descriptiva. Para establecer normalidad de los datos, se utilizó la prueba de Kolmogorov Smirnov (k-s). Para determinar la asociación del nivel de adecuación y género se utilizó la prueba de independencia de chi cuadrado (x2). Finalmente, para determinar la diferencia entre el total de porcentajes de niveles de adecuación se utilizaron los rangos de Wilcoxon con un valor para establecer significancia de $\mathrm{p}<0,05$.

Para la realización del presente estudio se contó con la aprobación mediante Resolución $\mathrm{N}^{\circ}$ 3227-2019-UNHEVAL-CU, emitida por la Universidad Nacional Hermilio Valdizán. Todos los participantes fueron informados acerca del estudio y firmaron el consentimiento informado.

\section{Resultados y discusión}

La muestra estuvo conformada por personas mayores de edad del género femenino $(\mathrm{N}=106$; $54,6 \%)$ y género masculino $(\mathrm{N}=88 ; 45,4 \%)$. La edad promedio entre los grupos fue de 34,0 $(5,0)$ años. La estatura promedio alcanzó $162 \mathrm{~cm}(5,9)$ en varones y $156 \mathrm{~cm}(5,1)$ en mujeres. El percentil más extremo de la distribución con respecto a la estatura alcanzó en varones $175,1 \mathrm{~cm}\left(\mathrm{p} 95^{\mathrm{th}}\right)$ y $150 \mathrm{~cm}$ (p 5th) en mujeres, por lo que existió una variabilidad de $25,1 \mathrm{~cm}$ entre ambos grupos. Los datos de todas las dimensiones siguieron una distribución normal medida con la prueba de K-s ( $>0,05)$. La dimensión con mayor dispersión fue la altura poplítea $(4,9)$ y el ancho de caderas $(4,7)$, mientras que en las mujeres la distancia glúteo poplítea $(3,1)$ (ver tabla 1$)$.

Con respecto al mobiliario, las dimensiones y medidas que se consideraron para el análisis, así como su descripción se encuentran resumidas en la figura 1. 
La tabla 2 muestra los resultados del estudio con respecto a las dimensiones antropométricas de los participantes y el nivel de adecuación. Las medidas de profundidad de asiento ( $\mathrm{x} 2=9,0 ; \mathrm{p}<0,05)$; altura de asiento $(\mathrm{x} 2=13,3 ; \mathrm{p}<0,05)$ y altura de respaldar $(\mathrm{x} 2=7,95$; $\mathrm{p}<0,05)$ se asocian significativamente al género de los participantes. Las medidas de profundidad del asiento y altura de respaldar se asociaron principalmente a los varones debido a la poca profundidad del asiento (82\%), y la corta altura del respaldo (45\%); mientras que en las mujeres se asoció principalmente a la elevada altura del asiento (83\%). La adecuación del ancho del asiento y la altura del tablero no fue independiente del género de los participantes. En ambos casos, la altura del tablero en más del 95\% estuvo demasiado alto. En más del 75\% de los casos la altura del asiento fue inadecuada. La figura 2, muestra esta problemática de manera gráfica.

El nivel de adecuación de los usuarios al mobiliario con porcentajes no adecuados es significativamente mayor para la población en general $(\mathrm{z}=-6,26 ; \mathrm{p}<0,001)$. Los varones $(\mathrm{z}=-$ 4,62; $\mathrm{p}<0,001)$ y las mujeres $(\mathrm{z}=-4,20 ; \mathrm{p}<0,001)$ tienen porcentajes significativamente mayores de no adecuación al mobiliario. Por lo que se concluye una no adecuación de los usuarios al mobiliario que excede el 60\% (ver figura 3).

Tabla 1. Perfil antropométrico de la población muestreada.

\begin{tabular}{|c|c|c|c|c|c|c|c|c|c|c|}
\hline \multirow{3}{*}{$\begin{array}{l}\text { Dimensiones } \\
\text { antropométricas }\end{array}$} & \multicolumn{5}{|c|}{$\operatorname{Varones}(\mathrm{N}=\mathbf{8 8})$} & \multicolumn{5}{|c|}{ Mujeres $(\mathrm{N}=106)$} \\
\hline & \multirow{2}{*}{ Media } & \multirow{2}{*}{ DE } & \multicolumn{3}{|c|}{ Percentiles } & \multirow{2}{*}{ Media } & \multirow{2}{*}{ DE } & \multicolumn{3}{|c|}{ Percentiles } \\
\hline & & & 5 & 50 & 95 & & & 5 & 50 & 95 \\
\hline Estatura parado* & 162,0 & 5,9 & 155,2 & 160,4 & 175,1 & 156,5 & 5,1 & 150,0 & 155,5 & 165,4 \\
\hline Ancho de Caderas* & $40, .8$ & 4,7 & 33,2 & 40,9 & 47,0 & 40,5 & 2,9 & 37,0 & 41,0 & 46,2 \\
\hline Distancia glúteo-poplítea* & 46,2 & 3,4 & 41,6 & 46,0 & 50,9 & 44,5 & 3,1 & 40,7 & 43,6 & 50,2 \\
\hline Altura poplítea* & 44,1 & 4,9 & 39,0 & 43,1 & 52,0 & 40,2 & 2,4 & 36,3 & 40,0 & 44,0 \\
\hline Codo asiento* & 24,8 & 2,3 & 21,3 & 24,7 & 28,0 & 24,0 & 2,1 & 21,0 & 24,0 & 28,0 \\
\hline Escápula-asiento* & 44,9 & 2,4 & 40,2 & 44,8 & 48,9 & 42,5 & 2,7 & 38,6 & 42,2 & 47,2 \\
\hline Muslo-asiento* & 13,5 & 2,4 & 9,5 & 13,7 & 17,0 & 12,9 & 1,7 & 10,0 & 13,0 & 15,7 \\
\hline
\end{tabular}

Nota. Medidas siguen una distribución normal (k-s ; p>0,05); medidas en centímetros $(\mathrm{cm})$. 
Tabla 2. Nivel de adecuación de las dimensiones antropométricas de participantes al mobiliario unipersonal con tablero acoplado.

\begin{tabular}{|c|c|c|c|c|c|c|c|c|c|c|c|c|}
\hline \multirow{2}{*}{$\begin{array}{l}\text { Dimensiones } \\
\text { del mobiliario }\end{array}$} & \multirow[b]{2}{*}{ Género } & \multirow{2}{*}{\multicolumn{2}{|c|}{\begin{tabular}{|c|} 
ADECUADO \\
$\begin{array}{c}\text { Total } \\
\text { adecuado }\end{array}$ \\
\end{tabular}}} & \multicolumn{8}{|c|}{ NO ADECUADO - DESAJUSTE } & \multirow[b]{2}{*}{$\mathbf{p}^{*}$} \\
\hline & & & & \multicolumn{2}{|c|}{$\begin{array}{l}\text { Excede } \\
\text { medida }\end{array}$} & \multicolumn{2}{|c|}{$\begin{array}{c}\text { Falta } \\
\text { medida }\end{array}$} & \multicolumn{4}{|c|}{ Total no adecuado } & \\
\hline \multirow{3}{*}{ Ancho del asiento ${ }^{\mathrm{a}}$} & Varones & 36 & $(41 \%)$ & 6 & $(7 \%$ & 46 & $(52 \%)$ & 52 & ( & $59 \%$ & ) & \multirow{3}{*}{0.35} \\
\hline & Mujeres & 56 & $(53 \%)$ & 10 & $(9 \%$ & 40 & $(38 \%)$ & 50 & ( & $47 \%$ & ) & \\
\hline & Total & 92 & $(47 \%)$ & 16 & $(8 \%)$ & 86 & $(44 \%)$ & 102 & ( & $53 \%$ & ) & \\
\hline \multirow{3}{*}{$\begin{array}{l}\text { Profundidad de } \\
\text { asiento }^{\mathrm{b}}\end{array}$} & & 16 & $(18 \%)$ & $\mathrm{O}$ & $(0 \%)$ & 72 & $(82 \%)$ & 72 & ( & $82 \%$ & ) & \multirow{3}{*}{$0.03^{* *}$} \\
\hline & Mujeres & 50 & $(47 \%)$ & $\mathrm{O}$ & $(0 \%$ & 56 & $(53 \%)$ & 56 & ( & $53 \%$ & ) & \\
\hline & Total & 66 & $(34 \%)$ & $\mathrm{O}$ & $(0 \%)$ & 128 & $(66 \%)$ & 128 & ( & $66 \%$ & ) & \\
\hline \multirow{3}{*}{ Altura de asiento ${ }^{c}$} & Varones & 30 & $(34 \%)$ & 46 & $(52 \%)$ & $\begin{array}{l}12 \\
\end{array}$ & $(14 \%)$ & 58 & ( & $66 \%$ & ) & \multirow{3}{*}{$0.001^{* *}$} \\
\hline & Mujeres & 18 & $(17 \%)$ & 88 & $(83 \%)$ & 0 & $(0 \%)$ & 88 & ( & $83 \%$ & ) & \\
\hline & Total & 48 & $(25 \%)$ & 134 & $(69 \%)$ & 12 & $(6 \%)$ & 146 & & $75 \%$ & ) & \\
\hline \multirow{3}{*}{ Altura de respaldar ${ }^{c}$} & Varones & 48 & $(55 \%)$ & 0 & $(0 \%)$ & 40 & $(45 \%)$ & 40 & ( & $45 \%$ & ) & \multirow{3}{*}{$0.001^{* *}$} \\
\hline & Mujeres & 86 & $(81 \%)$ & $\mathrm{O}$ & $(0 \%)$ & 20 & $(19 \%)$ & 20 & ( & $19 \%$ & ) & \\
\hline & Total & 134 & $(69 \%)$ & 0 & $(0 \%$ & 60 & $(31 \%)$ & 60 & ( & $31 \%$ & ) & \\
\hline \multirow{3}{*}{ Altura tablero $^{\mathrm{c}}$} & Varones & 4 & $(5 \%)$ & 84 & $(95 \%)$ & $\mathrm{O}$ & $(0 \%)$ & 84 & ( & $95 \%$ & ) & \multirow{3}{*}{0.45} \\
\hline & Mujeres & 2 & $(2 \%$ & 104 & $(98 \%)$ & 0 & ( $0 \%$ & 104 & ( & $98 \%$ & ) & \\
\hline & Total & 6 & $(3 \%)$ & 188 & $(97 \%)$ & $\mathrm{O}$ & $(0 \%)$ & 188 & & $97 \%$ & ) & \\
\hline
\end{tabular}

Nota. $\mathrm{P}=$ chi cuadrado de independencia; ${ }^{* *}$ diferencia significativa; $(\mathrm{a})$ excede medida $=$ muy ancho; falta medida = muy estrecho; $(b)$ excede medida $=$ muy profundo; falta medida $=$ poco profundo; $(\mathrm{c})$ excede medida $=$ muy alto; falta medida $=$ muy bajo.

\begin{tabular}{lccc}
\hline \multirow{2}{*}{ Dimension } & Medida & Mobiliario unipersonal \\
\cline { 2 - 4 } Ancho del asiento (A) & 44 & Representacion \\
\hline profundidad de asiento (B) & 35 & \\
Altura del asiento (C) & 47 & \\
Altura del respaldar (D) & 40 & B
\end{tabular}

Figura 1. Medidas del mobiliario unipersonal con tablero acoplado. Nota. Medidas en centímetros $(\mathrm{cm})$. 


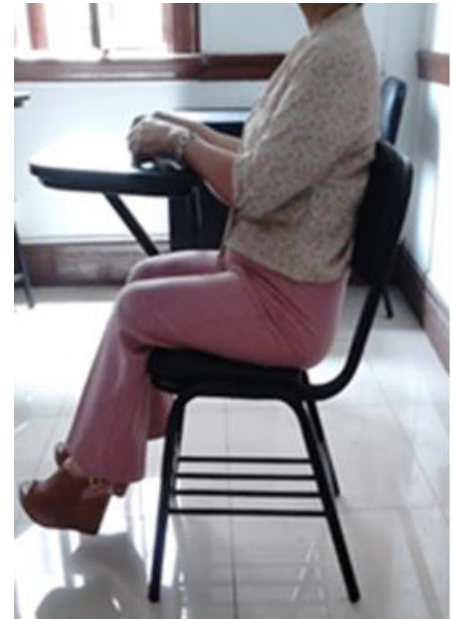

(a)

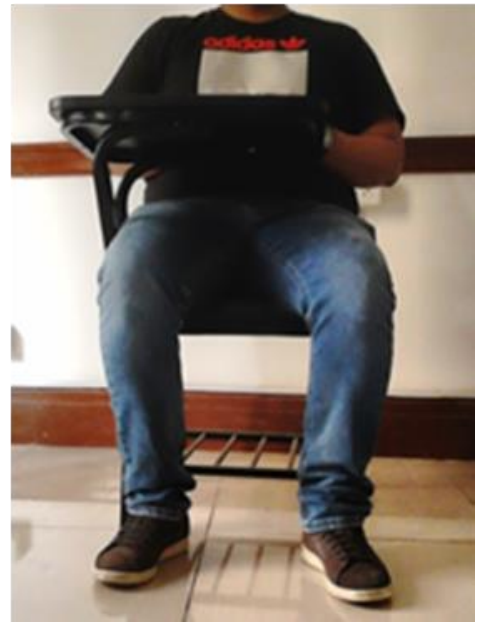

(b)

Figura 2. Problemas de adecuación del mobiliario observado en la muestra. Nota. Altura de asiento y tablero elevados para usuaria (a); ancho del asiento estrecho y poco profundo, además de altura de tablero elevado para usuario masculino (b).

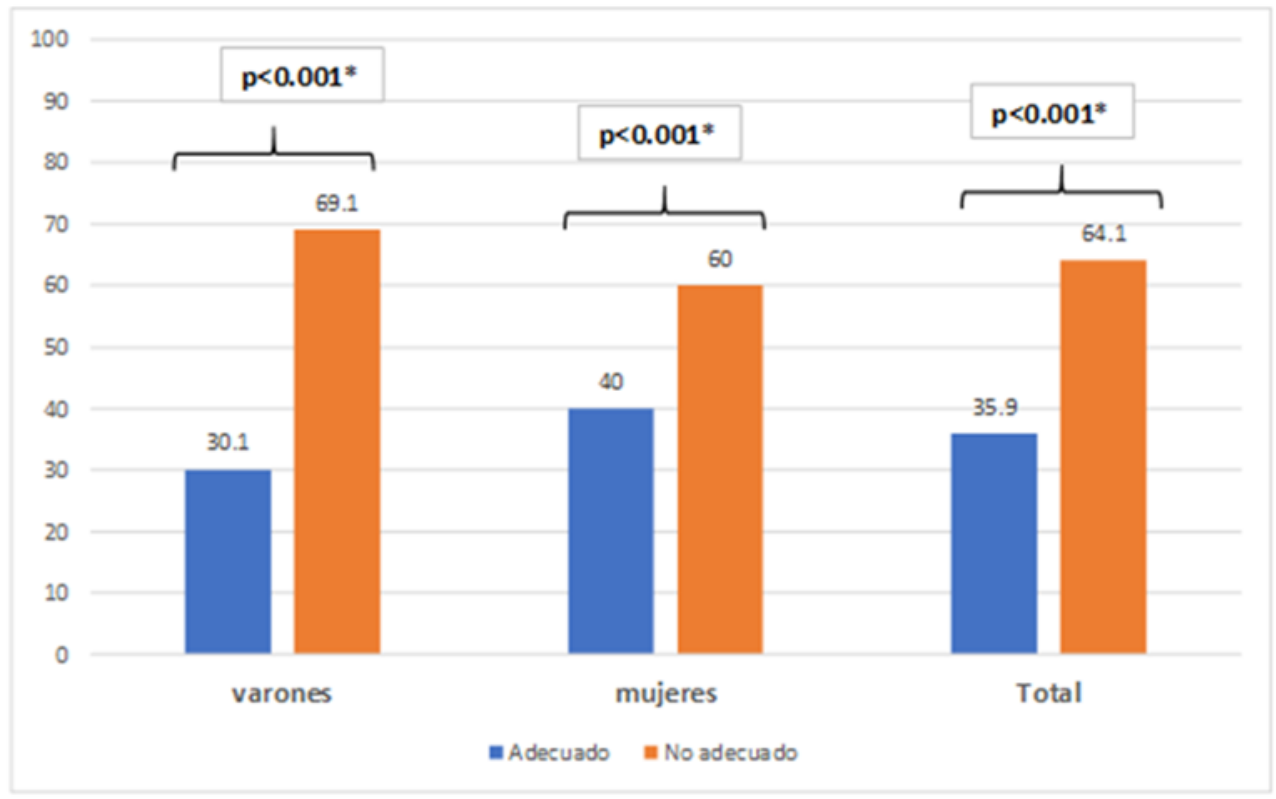

Figura 3. Porcentaje de adecuación final a los mobiliarios.

Los resultados del estudio antropométrico evidenció una estatura promedio en varones de $162,9 \mathrm{~cm}(5,9)$ y en mujeres de $156,5 \mathrm{~cm}(5,1)$, los cuales guardan similitud con los estudios recientes de estatura y genética de la población peruana (Asgari et al., 2019), excepto por la estatura de las mujeres en donde se evidenció un mayor promedio $(+3,6 \mathrm{~cm})$ en la muestra. De igual manera, en la región centro del país, Ramírez (2006) estableció un promedio inferior de estatura de 158,4 cm en varones, teniendo una diferencia de 4,5 cm con respecto al estudio, por lo que se evidencia una gran diversidad de las medidas en el país entre la población general y en el centro. Esta diversidad se ve reflejada en la población representada en el presente estudio. Además de la diversidad, los peruanos tienen una de las 
estaturas más bajas comparándola con la población mundial (NCD-RisC, 2016). Esta evidente diversidad y diferencia de estaturas entre varones y mujeres, refleja la enorme variabilidad de las medidas, las cuales son evidenciadas, por ejemplo, por la gran desviación estándar de las medidas de altura, como la del codo al asiento o poplítea. Esto denota la gran dificultad de los participantes en adecuarse a los actuales mobiliarios y el gran reto de diseñar para el 90\% de la población (Pheasant \& Haslegrave, 2006)

Una de las medidas que presentó mayor variación entre ambos grupos fue la altura poplítea de las mujeres $(4 \mathrm{~cm})$, la cual se ve reflejada en una evidente discrepancia en la altura del asiento con respecto a los varones. Para el 83\% de las mujeres, la altura del asiento era muy alta; como consecuencia puede existir un aumento de la presión en la zona posterior de los muslos, creando incomodidad y adormecimiento. Para compensar esta discrepancia, las personas pueden verse obligadas a tener que deslizar sus glúteos hacia el borde del asiento, extendiendo el tronco para apoyarse en el respaldo, llevando a una falta de apoyo de la región lumbar, y por tanto, cambiar la curvatura fisiológica de la columna, así como el centro de gravedad aumentando zonas de presión (Chaffin et al., 2006). Desde el punto de vista del desempeño, la presión e incomodidad, así como la falta de apoyo de los pies, podría aumentar los niveles de distracción, debido a la reducción de la retroalimentación propioceptiva para dar estabilidad al segmento inferior pudiendo perjudicar de algún modo su atención a la clase. Estos hallazgos se asemejan a la de otras investigaciones que abordaron el mobiliario escolar, quienes también encontraron esta discordancia, lo cual pone en riesgo la salud de estudiantes en general (Castellucci et al., 2010, Gutiérrez \& Apud, 1992, Hoque et al., 2014). Como recomendación se debería ajustar la altura de los asientos a los de menor altura poplítea, ya que los de mayor distancia se acomodan más fácilmente que aquellos cuya altura poplítea es inferior a la del asiento, caso contrario deberían usar reposapiés o algún sistema ajustable (Thariq et al., 2010).

El asiento resultó ser muy corto de profundidad, debido a que las mujeres, quienes presentaron el menor percentil de la población, tienen mayor adecuación que los varones quienes son más altos, y por tanto, su nivel de adecuación principalmente es por poca profundidad. Esto puede ocasionar un apoyo insuficiente de los muslos, además de incrementar la presión sobre las tuberosidades isquiáticas y la parte posterior de los muslos, que sumado a la altura elevada del asiento puede aumentar la presión. Estos resultados difieren con otros estudios en donde la profundidad fue mayor, creando un desajuste por exceso de medida (Hoque et al., 2014, Parcells et al., 1999). Por tanto, se observa que los criterios de diseño no siguen una armonía proporcional, además de no estar centrados en el usuario. Otros estudios no han considerado este desajuste como un aspecto relevante en sus trabajos, lo cual puede limitar en cierta manera la mejora del diseño del mobiliario universitario (Párraga-Velásquez \& García-Zapata, 2014). En el estudio no se evidencia ningún mobiliario que sea muy profundo, sin embargo, es evidente que en usuarios más pequeños puedan ocurrir discrepancia por exceso de medida.

En lo referente al tablero, se evidenció una altura muy elevada con respecto a la medida codo-asiento en ambos géneros (96,9\%), similar a otros estudios (Cadavid, 2007; Castellucci et al., 2010). Esta elevación de altura incrementa la contracción de los músculos del trapecio (fibras superiores) y estimula la abducción de los hombros por encima de los $25^{\circ}$ de confort, 
lo cual podría generar dolor e incomodidad (Grandjean \& Kroemer, 1995). Además, se debe considerar que los mobiliarios evaluados fueron principalmente utilizados para clases teóricas, más no para actividades prácticas, tales como diseño, computación o arquitectura en donde las características del tablero probablemente requerirían otro tipo de características y referencias.

Un poco más de la mitad de los participantes tuvo problemas de adecuación con respecto al ancho del asiento, sin embargo los datos no establecen una diferencia estadística entre los valores finales y el género. La discrepancia en el ancho, principalmente, fue por falta de medida, lo cual puede causar que los participantes tengan dificultades para sostener una postura estable. Al ser el asiento estrecho, puede generar falta de apoyo sobre los glúteos y falta de ajuste. Con respecto al respaldo, las mujeres encontraron un mejor ajuste que los varones, debido a su menor estatura y a la baja altura del respaldo, sin embargo, para los varones resultó ser muy bajo, no permitiendo un buen apoyo que reduzca una posible sobrecarga postural producto del tiempo prolongado.

\section{Conclusiones}

En general, la carpeta unipersonal con tablero acoplado presenta problemas de armonía proporcional, porque sus medidas excedieron en la altura del asiento y del tablero, faltaron medidas en la profundidad del asiento, y el respaldo resultó ser corto. Esto denota la falta de criterios de diseño ergonómico, necesarios y requeridos para asegurar el bienestar de los usuarios. Por tanto, urge establecer criterios para el diseño de mobiliario universitario, con especificaciones técnicas y claras que salvaguarden el bienestar de los estudiantes universitarios. Actualmente, en Perú se encuentran disponibles normativas técnicas para la adquisición de mobiliario escolar sólo para los niveles de inicial, primaria y secundaria, por lo que el presente estudio crea un precedente para valorar la importancia de la variable ergonómica en el diseño de mobiliarios para prevenir posibles MME que puedan desencadenar en otros problemas que afecten el bienestar de los estudiantes universitarios y su desempeño en clase. Además, establece un parámetro referencial único que puede facilitar el diseño de mobiliario. Sin embargo, es necesario el compromiso de autoridades e instituciones para crear futuras normas con estudios técnicos, precisos y validados, que aseguren un mobiliario ergonómico, de tal forma, que se puedan establecer parámetros legales que se ajusten a la realidad del país y que sean de aplicación obligatoria.

Como recomendación principal, se deberían ajustar la altura de los asientos a los de menor percentil, tomando en cuenta la naturaleza de la tarea a realizar en el mobiliario. Con respecto al tablero, este debería dar buen apoyo para el material de lectura, el cual requiere de una ligera elevación (hasta $+5 \mathrm{~cm}$ ) e inclinación $\left(5^{\circ}-10^{\circ}\right)$ para facilitar la visualización, sin embargo esta elevación no debe ser muy pronunciada porque incrementaría la carga en los segmentos superiores. Además, se recomienda un diseño en " $U$ ” para las personas con mayor volumen abdominal, por lo que podría considerarse en futuros diseños.

Se recomienda además efectuar estudios antropométricos con una mayor muestra para crear estándares nacionales validados, que permitan estimar parámetros referenciales 
necesarios para lograr diseños ergonómicos. Además, se deberían ampliar estudios donde se consideren otras variables, como la calidad del material, diferentes tipos de mobiliarios, tiempo de permanencia sentado, su uso, su relación con el rendimiento académico, así como su impacto en poblaciones mayores.

Finalmente, se concluye que existe un evidente desajuste entre las medidas antropométricas de los estudiantes y el mobiliario unipersonal con tablero acoplado, existiendo grandes problemas de adecuación con respecto a la altura poplítea, altura del tablero, y distancia glúteo poplítea, principalmente. Este resultado evidencia una primera aproximación en el Perú para el diseño de mobiliarios universitarios, en donde la variable ergonómica debe ser considerada para la mejora del bienestar de los estudiantes y su desempeño en clases.

\section{Referencias}

Asgari, S., Luo, Y., Belbin, G. M., Bartell, E., Roger, R., Slowikowski, K., Contreras, C., Yataco, R., Galea, J. T., Jimenez, J., Coit, J. M., Farronay, C., Nazarian, R. M., O Connor, T. D., Dietz, H. C., Hirschhorn, J., Guio, H., Lecca, L., Kenny, E. E., ... Raychaudhuri, S. (2019). A positively selected, common, missense variant in FBN1 confers a 2.2 centimeter reduction of height in the Peruvian population. bioRxiv, 561241. https://doi.org/10.1101/561241

Ávila, R., Prado, L., \& González, E. (2001). Dimensiones antropométricas de población latinoamericana: México, Cuba, Colombia y Chile. Universidad de Guadalajara.

Cadavid, G. A. S. (2007). Evaluación dimensional entre el mobiliario escolar NTC 4734 y la población escolar del Colegio San Juan Eudes. Medellín-Colombia 2005. Iconofacto, 3(4), 100-105. https://dialnet.unirioja.es/descarga/articulo/5204380.pdf

Castellucci, H., Arezes, P., \& Viviani, C. (2010). Mismatch between classroom furniture and anthropometric measures in Chilean schools. Applied ergonomics, 41(4), 563-568. https://pubmed.ncbi.nlm.nih.gov/20031115

Castellucci, H. I., Viviani, C. A., Molenbroek, J. F. M., Arezes, P. M., Martínez, M., Aparici, V., \& Bragança, S. (2019). Anthropometric characteristics of Chilean workers for ergonomic and $\begin{array}{llll}\text { design } & \text { purposes. } & \text { 62(3), } & \text { 459-474. }\end{array}$ https://doi.org/10.1080/00140139.2018.1540725

Chaffin, D. B., Andersson, G. B., \& Martin, B. J. (2006). Guidelines for work in sitting posture. En Occupational biomechanics. John Wiley \& Sons.

Falzon, P. (2009). Manual de Ergonomia (1.a ed.). Modus Laborandi.

Grandjean, E., \& Kroemer K. (1995). Fitting task to he Human (4 th). CRC Press.

Gutiérrez, H., \& Apud, S. (1992). Estudio antropométrico y criterios ergonómicos para la evaluación y el diseño de mobiliario escolar. Cuad. méd.-soc. (Santiago de Chile), 33(4), 728o. https://dialnet.unirioja.es/descarga/articulo/4835614.pdf 
Hoque, A., Parvez, M., Halder, P., \& Szecsi, T. (2014). Ergonomic design of classroom furniture for university students of Bangladesh. Journal of Industrial and Production Engineering, 31(5), 239-252. https://doi.org/10.1080/21681015.2014.940069

ISO (2012). ISO 15535:2012 General requirements for establishing anthropometric databases.

ISO (2017). ISO 7250-1:2017 Basic human body measurements for technological design-Part 1: Body measurement definitions and landmarks.

NCD Risk Factor Collaboration [NCD-RisC] (2016). A century of trends in adult human height. eLife, 5, e13410. https://doi.org/10.7554/eLife.13410

Normas Técnicas Peruanas sobre muebles, tecnología de la información, tortas de semillas

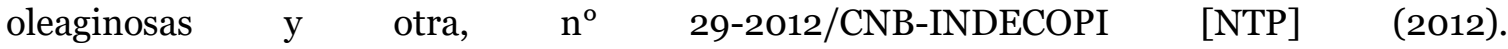
https://busquedas.elperuano.pe/download/url/aprueban-cargo-de-destino-de-gerente-deadministracion-y-fin-resolucion-n-067-2012-servir-pe-787165-1

Norton, K., Whittingham, N., Carter, L., Kerr, D., Gore, C., \& Marfell-Jones, M. (2000). Técnicas de medición en antropometría. En: Norton, K., Olds, T. (eds.). Antropométrica. Biosystem.

Parcells, C., Stommel, M., \& Hubbard, R. P. (1999). Mismatch of classroom furniture and student body dimensions: Empirical findings and health implications. Journal of Adolescent Health, 24(4), 265-273. https://doi.org/10.1016/S1054-139X(98)o0113-X

Párraga-Velásquez, R. \& García-Zapata, T. (2014). Diseño ergonómico de aulas universitarias que permitan optimizar el confort y reducir la fatiga de estudiantes y docentes. Industrial Data, 17(2), 7-16. https://www.redalyc.org/pdf/816/81640856002.pdf

Parvez, M. S., Halder, P. K., \& Szecsi, T. (2014). Ergonomic design of classroom furniture for university students of Bangladesh AU - Hoque, A.S.M. Journal of Industrial and Production Engineering, 31(5), 239-252. https://doi.org/10.1080/21681015.2014.940069

Pheasant, S., \& Haslegrave, C. M. (2006a). Principles and practice of anthropometrics. En Bodyspace: Anthropometry, ergonomics and the design of work (Third Edition). CRC Press.

Pheasant, S., \& Haslegrave, C. M. (2006b). Working design. En Bodyspace: Anthropometry, ergonomics and the design of work (Third Edition). CRC Press.

Ramírez, A. V. (2006). Antropometría del trabajador minero de la altura. Anales de la Facultad de Medicina, 67, 298-309. http://www.scielo.org.pe/scielo.php?script=sci arttext\&pid=S1025-55832006000400004

Sistema Nacional de Evaluación Acreditación y Certificación de la Calidad Educativa [SINEACE] (2016). Modelo de Acreditación para Programas de Estudios de Educación Superior Universitaria (p. 16). https://www.sineace.gob.pe/wp-content/uploads/2014/o8/Anexo-1nuevo-modelo-programas-Resolucion-175.pdf

Thariq, M. M., Munasinghe, H., \& Abeysekara, J. (2010). Designing chairs with mounted desktop for university students: Ergonomics and comfort. International Journal of Industrial Ergonomics, 40(1), 8-18. https://doi.org/10.1016/j.ergon.2009.10.003 